\title{
WILEY-VCH
}

DOI: $10.1002 /$ ((please add manuscript number))

Article type: Full Paper

\section{Highly tunable polarized chromatic plasmonic films based on sub- wavelength grating templates}

Jun Zheng*, Zhi-Cheng Ye, Cheng-Liang Wang, Yi-Fei Fu, Xin-Ran Huang and Zheng-Ming Sheng

Jun Zheng and Xin-Ran Huang

Key Laboratory for Laser Plasmas (Ministry of Education), School of Physics and astronomy, and Collaborative Innovation Center of IFSA (CICIFSA), Shanghai Jiao Tong University, Shanghai, 200240, China

E-mail: jzheng@sjtu.edu.cn

Zhi-Cheng Ye, Cheng-Liang Wang, and Yi-Fei Fu

Department of Electronic Engineering, Shanghai Jiao Tong University, Shanghai, 200240,

China

Zheng-Ming Sheng

SUPA and Department of Physics, University of Strathclyde, Glasgow G4 0NG, UK

Key Laboratory for Laser Plasmas (Ministry of Education), School of Physics and astronomy, and Collaborative Innovation Center of IFSA (CICIFSA), Shanghai Jiao Tong University, Shanghai, 200240, China

Keywords: plasmonic structural colors, sub-wavelength grating, metallic nanowire gratings, polarizer, color filter

A kind of polarized chromatic plasmonic film is proposed based on sub-wavelength grating structure, which enables 'blue transmission' for the transverse electric (TE) light and 'red transmission' for the transverse magnetic (TM) light. Metal-insulator-metal plasmonic waveguiding and metallic nanowire scattering are revealed to be responsible for the chromatic shift. Based upon the unique transmission spectrum characteristics of such films, polarized chromatic plasmonic tags (PCPTs) can be flexibly fabricated by patterning dielectric grating templates with designed figures and depositing appropriate thickness of metal. These PCPTs, simultaneously possessing directly visible unpolarized transmission colors and concealed distinct polarization-dependent color shift, can be widely used as anti-counterfeiting tags with higher security than the diffractive types of holograms. 


\section{WILEY-VCH}

\section{Introduction}

Metallic nanoarray based plasmonic structutral color (PSC) has been widely investigated as colorfilters, ${ }^{[1-3]}$ polarizers, ${ }^{[4-6]}$ and highly sensitive surface plasmon resonance sensors. ${ }^{[7]}$ The devices based on PSC have the advantages of longer lifetime, thinner dimension, better performance than the conventional types, which can be widely used in displays, anticounterfeiting, ${ }^{[8,9]}$ and image sensors. ${ }^{[10]}$ Generally, the functions of metallic nanoarrays are realized by the following three mechanisms: (1) the localized surface plasmon resonance in metallic nano-units, which leads to absorption dips in the transmission or reflection; ${ }^{[1,12]}(2)$ the surface plasmon spoofed by the nanoarrays, which lead to extraordinary transmission through dielectric apertures below diffraction limits; ${ }^{[13,14]}(3)$ the plasmonic waveguiding of dielectric slits, which supports broadband polarized transmission of TM light, while prohibits the propagation of TE light. ${ }^{[15]}$ Utilizing these mechanisms, the spectra of metallic nanoarray can be flexibly tuned to meet a variety of applications by engineering the metal thickness, profile of the unit cells, and the period. For example, by tailoring the profile of the nano unit cell to be asymmetric (linear, elliptical, or rectangular), polarization-dependent tunability can be imposed on the PSC to make integrated colorfilters and polarizers ${ }^{[16,17]}$ for more compact and lower-power-consumption liquid crystal displays (LCDs). Recently, actively tunable PSCs based on elastomeric substrates have been reported. By stretching the substrates, the pitch and therefore scattering colors of PSCs can be modified in a wide range, showing great promising as novel display devices. ${ }^{[18]}$ Besides the structure, the permittivity of the metal materials also strongly affects the spectrum characteristics. For instance, with small damping factors, gold and silver metallic nanoarrays have sharp surface plasmon resonance dips that suitable for high figure of merit bio-sensing. ${ }^{[19]}$ While aluminium (Al) has a large absolute value of complex permittivity and a large plasmon frequency to maintain plasmonic characteristics at wavelength down to ultraviolet, thus it is suitable to make broadband 


\section{WILEY-VCH}

nanowire polarizers with high extinction ratio of TM over TE light in the transmission. In addition, $\mathrm{Al}$ has been widely used in the packaging, decoration, and laser hologram tags to enhance the reflection of diffraction gratings. ${ }^{[19-21]}$

In this work, we propose a kind of transmission films of one-dimensional metallic nanowire gratings (MNGs), which show polarization-dependent PSC over broad spectrum. Such films can be used as a new type of high-security and more recognizable anti-counterfeiting tags: polarized chromatic plasmonic tags (PCPTs). The physical mechanisms responsible for the polarization-dependent chromaticity are revealed, which are used in the device design. In Section. 2, the fabrication of the films and tags is presented and their transmission spectra are described as a function of the MNG parameters and incident light parameters. In Section 3, a physical model is presented to decribe the polarization-dependent chromaticity. The paper concludes with a summary in Section 4.

\section{Device fabrication and spectra measurements}

\subsection{Device fabrication and basic principle}

As shown in Figure 1, the fabrication procedure of MNG-based PCPT includes three steps. Firstly, dielectric grating templates are fabricated by using laser interference lithography $(\mathrm{LIL})^{[22]}$ or nano-imprinting lithography (NIL), which have shown the advantages in the mass production of MNGs. Then, the figures of tags are patterned on the templates by using flexible mask-free laser engraving or rapid mask-based UV printing. Finally, by depositing appropriate thickness of $\mathrm{Al}$, the demanded colors of the plasmonic tags are obtained, where only gratings in the figures of interest are remained. The diffractive snapshots of the samples fabricated in each step are presented in Figure 1(a3), (b3), and (c3). 


\section{WILEY-VCH}

A schematic diagram of the MNG forming the PCPTs is illustrated in Figure 2(a). The device includes three layers of gratings: top Al-air, middle photoresist (PR) bar (coated with a thin layer of $\mathrm{Al}$ on the sidewalls)-air, and bottom PR-Al gratings. A collimated white light beam is incident to the device with angle of $\theta$. The incident plane is perpendicular to the grating lines. The TE light with shorter wavelength and TM light with longer wavelength are transmitted through the film as shown by the colored arrows. The scanning electronic microscopy (SEM) images of the fabricated PCPT are shown in Figure 2(b), where the grating was fabricated by using NIL on a glass substrate with pitch $T=180 \mathrm{~nm}$, width of the PR $t_{1}=120 \mathrm{~nm}$, and thickness of PR $h_{2}=130 \mathrm{~nm}$. After the patterning of the logo of Shanghai Jiao Tong Universtiy by using laser engraving, $\mathrm{Al}$ of $10 \mathrm{~nm}$ in thickness was conformally deposited on the dielectric grating.

As displayed in Figure 2(c), the measured transmittance of TE and TM lights have a peak in the 'blue' region with wavelength of $420 \mathrm{~nm}$ (blue line) and 'red' region with wavelength of $770 \mathrm{~nm}$ (red line), respectively. More specifically, the measured TM transmittance $T_{T M}$ is $1.2 \%$ at wavelength $420 \mathrm{~nm}$ and $29 \%$ at wavelength $770 \mathrm{~nm}$, while the transmittance of TE light $T_{T E}$ at these two wavelengths are $32 \%$ and $3.8 \%$, respectively. It means the extinction ratio $T_{T E} / T_{T M}$ is about 27 at wavelength $420 \mathrm{~nm}$, and the extinction ratio $T_{T M} / T_{T E}$ is about 8 at wavelength $770 \mathrm{~nm}$. Due to the high contrast ratios between the peaks and valleys of the two orthogonal polarizations, noticable polarization-dependent color shifts can be obtained.

The measured polarization-dependent transmitted spectra are shown in Figure 2(c), which can be represented by $T(\lambda, \varphi)=T_{T M}(\lambda) \cos ^{2} \varphi+T_{T E}(\lambda) \sin ^{2} \varphi$, where $\lambda$ is the wavelength of light in free space and $\varphi$ is the cross angle between the electric vector with the incident plane. It is $\varphi=$ $0^{\circ}$ for $\mathrm{TM}$ polarization and $90^{\circ}$ for TE polarization. The corresponding chromaticity mapped as red points in the CIE 1931 xy chromaticity diagram in Figure 2(d) further illustrates the 


\section{WILEY-VCH}

distinct color shifts. The snapshots of the PCPT are shown in Figure 2(e), where the color shifts from golden to blue are vividly displayed as the polarization of the incident white light is rotated from $\operatorname{TM}\left(\varphi=0^{\circ}\right)$ to $\operatorname{TE}\left(\varphi=90^{\circ}\right)$. The video of the transmission can be found in the supplementary information of this paper. One may note that the color for the case of $\varphi=45^{\circ}$ is the same with that produced by unpolarized light, which means that decorative colorful pictures (purple in this case) can be directly observed without polarizers.

\subsection{Characterization of the transmission spectra}

As shown in Figure 2(c), there is a intersection point wavelength $\lambda_{c}$, where $T_{T M}\left(\lambda_{c}\right)=T_{T E}\left(\lambda_{c}\right)$. This can be used to evaluate the color difference between TE and TM lights, i.e., TE transmittance is higher than that of TM for $\lambda<\lambda_{c}$, and TM transmittance is higher than TE for $\lambda>\lambda_{c}$. Thus the 'blue' component in the transmitted color is mainly determined by TE light, and the 'red' part is mainly decided by TM light. In Figure 3(a), $\lambda_{c}$ is showed as a function of Al thickness $h_{1}$ and grating pitch $T$, calculated by using RSOFT and DiffractMOD ${ }^{\mathrm{TM}}$. Generally, with the increase of $h_{1}$ or decrease of $T, \lambda_{c}$ moves to shorter wavelengths, leading to the blue shift of the peaks for TE light and the broadening of the spectra for TM light. Besides the shift of $\lambda_{c}$, as shown in Figure 3(b), the TE transmission is weakened and the TM transmission at the shorter wavelengths is enhanced when the thickness of the Al layer is increased. This is due to the increasing inhibition and enhancement effects to TE and TM light, respectively, via plasmonic waveguiding. ${ }^{[23]}$ For Al thickness $h_{l}$ of $80 \mathrm{~nm}$, the transmission of TM light displays a white color with spectra entirely covering the visible lights, while the TE light exihibits black due to the weak transmittance of less than $0.01 \%$. The giant change of the transmitted colors from chromtic to black and white with the increasing Al thickness well illustrates the high tunability of the MNG-based PSC. 


\section{WILEY-VCH}

The schematic diagram and experimental results for the grating with Al thickness of $60 \mathrm{~nm}$ are shown in Figure 4. The transmitted spectra conspicuously show broadband polarization features with extinction ratio of $T_{T M} / T_{T E}$ reaching $40 \sim 140$ in the whole visible lights. The images of the characters 'SJTU' composed of thick-Al MNG in Figure 4(b) further display bright and dark transmission for TM and TE light, respectively.

A variety of 'blue' TE and 'red' TM transmission of PCPTs with different Al thickness and grating pitch are shown in Figure 5, which can display the high tunability of the chromatic characteristics of MNG-based PSC. At first, photoresist gratings with pitches of 350, 300 and $260 \mathrm{~nm}$ are fabricated on BK7 glass substrates as templates by using LIL. Then the templates are patterned with figures by using UV-printing, where only gratings are remained in the logos. Finally, a layer of Al film is deposited on the logos to make PCPTs. In LIL, the corresponding cross angles between the two laser beams (He-Cd laser, wavelength $442 \mathrm{~nm}$, KIMMON) are of $78^{\circ}, 95^{\circ}$, and $116^{\circ}$, respectively.

As shown by the measured spectra for $T=350 \mathrm{~nm}$ with $h_{l}=15$ and $40 \mathrm{~nm}$ in Figures 5(a1) and 5(a2), respectively, $\lambda_{c}$ is blue-shifted with increasing thickness of Al film. Moreover, their shifts of chromaticity with the incident light polarizations behave distinctly different, as mapped in Figure 2(d). The transmitted color and $\lambda_{c}$ can also be adjusted by the grating pitch as shown in Figures 5(a2-a4) for $T=350,300$ and $260 \mathrm{~nm}$. The shorter the pitch, the shorter the wavelength $\lambda_{c}$, in agreement with the simulation results in Figure 3(a). The corresponding simulated spectra and colors for TM and TE cases are presented with dashed lines in Figure 5(a) and circle markers in Figure 2(d) for comparison. The simulated transmittance is about twice of the experimental results. A series of experimental snapshots in Figure 5(b) vividly show the variations of the logo colors under different Al thickness and grating pitch. The pictures in the dashed rectangle are the same with those observed under unpolarized white 


\section{WILEY-VCH}

light. Related with this figure, four videos showing the light transmission are given in the supplementary information. In general, by changing the thickness of the metal layer and the grating pitch, preferable colors, as well as the color shift tendency with light polarization, are achievable.

\section{Analysis of the physical mechanisms}

Single nanowire resonant scattering, ${ }^{[24]}$ mutual nano-gap coupling ${ }^{[25]}$ and waveguiding of metal-insulator-metal nano-slits ${ }^{[26,27]}$ have been commonly known to be the main mechanisms determining the spectral characteristics of MNGs. However, the phenomena of 'blue' TE and 'red' TM- polartized chromatic tranmission found in this work have not been investigated. To understand how the plasmonic effects lead to such a large optical anisotropy, we plot the dispersion curves of the slit modes of the three layers of the MNGs in Figure 6 based upon our previous model ${ }^{[26]}$. Here the blue stars and red dots represent the real $\left(k_{z r}\right)$ and imaginary ( $\left.k_{z i}\right)$ parts of the wave number $k_{z}$, respectively. From these curves we can deduce: (1) Due to the negligible imaginary parts $k_{z i}$ of the wavenumber, TM light can pass through the top and bottom Al grating layer in plasmonic waveguide modes beyond diffraction limit. (2) The TE light in the top and bottom Al grating layers is lossy because $k_{z i}>>k_{z r}$. The larger the wavelength, the higher lossy the TE light, because of the increasing $k_{z i}$ with wavelength.

Besides the plasmonic waveguiding effect, the inherent scattering of metallic nanowires cannot be ignored. The forward scattering cross-sections $\left(S_{f}\right)$ of the MNGs with pitch of 180 $\mathrm{nm}$ are shown in Figure 7, which is calculated by LUMERICAL, FDTD Solutions ${ }^{\mathrm{TM}}$. For $h_{l}=$ $10 \mathrm{~nm}$ in Figure 7(a), $S_{f}$ shows obvious wavelength dependence for both polarizations. $S_{f}$ of TE light at wavelengths shorter than $\lambda_{c}$ is larger than that of TM light. $S_{f}$ of TM light at wavelengths longer than $\lambda_{c}$ is larger than that of TE light. For $h_{l}=60 \mathrm{~nm}$ in Figure 7(b), the 


\section{WILEY-VCH}

wavelength dependence of $S_{f}$ for TM light fades. But the value of $S_{f}$ is generally increased.

The wavelength dependence of $S_{f}$ for TE light is kept, while it is less than that of TM light in the whole range of visible lights, especially at the longer wavelengths.

Combining both plasmonic waveguiding and scattering effects, we can conclude: (1) In the thin metal case, TE light with shorter wavelength can still penetrate into the top and bottom layer of metallic gratings in the form of evanescent wave, while TM light with the same wavelengths suffers from metallic nanowire scattering as shown in Figure 7(a). Thus TE light has higher transmittance than TM light at the shorter wavelength; (2) With the increase of Al thickness, the plasmonic waveguiding effect gradually dominates, thus TE light only with much shorter wavelength can penetrate into the metallic gratings, while the TM light can transmit efficiently.

The transmittance can also be tuned by varying the thickness of the middle dielectric layer based on the well-known Fabri-Perrot (F-P) resonance effect, as shown by the transmission for wavelength $400 \mathrm{~nm}$ in Figure 8. The main inherent difference between the TM and TE polarizations caused by the waveguide effect is asssociated with the effective refractive index. The real part of effective refractive indexes $n_{\text {eff }}$ in the three layers have the relations $n_{\text {eff_top }}<$ $n_{\text {eff_mid }}<n_{\text {eff_bot }}$ for TM light and $n_{\text {eff_mid }}>n_{\text {eff_top }}, n_{\text {eff_bot }}$ for TE light, according to the dispersion relations shown in Figure 6. It means that there is a phase difference of $\pi$ between TM and TE caused by the interface reflectance. Thus the resonant wavelengths of the peaks for both polarizations are different. Although F-P effect can effectively tune the transmitance, there is always $T_{T E}$ (dashed blue line) $>T_{T M}$ (dashed voilet line) for small $h_{2}$ and $T_{T E}$ (solid blue line) $<T_{T M}$ (solid voilet line) for large $h_{2}$. Obviously, the 'blue' TE and 'Red' TM polarized chromatic transmission is mainly decided by the metallic thickness $h_{I}$ other than by the dielectric thickness $h_{2}$. The different effects of metal thickness and dielectric grating height to the spectra properties of MNGs help to break through the restriction in designing 
PCPTs where the transmittance and colors are shifted simultaneously by only tuning the metal thickness.

\section{Summary}

We have proposed a type of MNG films, which enables to show directly colors under the irradiation of unpolarized ambient white light. Moreover, it can display polarizationdependent color shifts. Plasmonic waveguiding of the slits and scattering of the metallic nanowries are revealed to be responsible for the characteristics of 'blue' TE and 'red' TM polarized transmission. The MNG-based PCPT has the following characteristics: (1) The grating pitch is sub-wavelength-scale, which can efficiently prevent unauthorized duplicating by using laser writing technology that micrometer-scaled hologram tags suffer from; (2) The transmitted colors can be flexibly tuned by engineering the metal thickness or grating pitch. (3) The transmitted colors deduced by plasmonic effect is distinctly different from the angledependent diffraction of holograms; (4) Even further, the concealed polarized chromaticity can significantly raise the security level of anti-counterfeiting tags.

The massive fabrication of such MNG film based PCPTs has been achieved. The procedure includes: sub-wavelength grating templates fabrication by NIL and LIL, figure patterning by laser engraving or UV-printing, and PSC engineering by depositing appropriate thickness of Al. The MNG-PCPTs can also be produced flexibly with demanded colors. Possessing the advantages of dramatic polarized color shift, much finer structure and compatible technology with present industrial capabilities, MNG-PCPTs are deemed to be readily applicable in the market of commercial tags, which have much higher securiety than the current diffractive hologram tags. 


\section{WILEY-VCH}

\section{Supporting Information}

Supporting Information is available from the Wiley Online Library or from the author.

\section{Acknowledgements}

This work was supported by the National Natural Science Foundation of China (Nos.

61775136 and 11721091)

Received: ((will be filled in by the editorial staff))

Revised: ((will be filled in by the editorial staff))

Published online: ((will be filled in by the editorial staff))

\section{References}

[1] Q. Chen and D. R. S. Cumming, Opt. Express 2010, 18, 14056.

[2] H. S. Lee, Y. T. Yoon, S. S. Lee, S. H. Kim and K. D. Lee, Opt. Express 2007, 15, 15457.

[3] Y. Liang, W. Peng, M. Lu and S. Chu, Opt. Express 2015, 23, 14434.

[4] Z. Yu, P. Deshpande, W. Wu, J. Wang and S. Y. Chou, Appl. Phys. Lett. 2000, 77, 927.

[5] X. P. Zhang, H. Liu, J. Tian, Y. Song and L. Wang, Nano Lett. 2008, 8, 2653.

[6] T. Weber, T. Käsebier, A. Szeghalmi, M. Knez, E. Kley and A. Tünnermann, Nanoscale Res. Lett. 2011, 6, 558.

[7] J. N. Anker, W. P. Hall, O. Lyandres, N. C. Shah, J. Zhao and R. P. V. Duyne, Nat. Mater. 2008, 7, 442.

[8] J. Sauvage-Vincent, S. Tonchev, C. Veillas, S. Reynaud and Y. Jourlin, Europ. Opt. Soc. Rap. Public. 2013, 8, 13015.

[9] E. L. Prime and D. H. Solomon, Angew. Chem. Int. Ed. 2010, 49, 3726.

[10] S. Yokogawa, S. P. Burgos and H. A. Atwater, Nano Lett. 2012, 12, 4349.

[11] E. Hutter and J. H. Fendler, Adv. Mater. 2012, 16, 1685.

[12] K. A. Willets, A. J. Wilson, V. Sundaresan and P. B. Joshi, Chem. Rev. 2017, 117, 7538. 


\section{WILEY-VCH}

[13] K. A. Willets, A. J. Wilson, V. Sundaresan and P. B. C. Genet and T. Ebbesen, Nature 2007, 445, 39 .

[14] N. L. Sun, J. Cui, Y. She, L. Lu, J. Zheng and Z. C. Ye, Opt. Mater. Express 2015, 5, 912.

[15] J. Zheng, Z. C. Ye, N. L. Sun, R. Zhang, Z. M. Sheng, H. P. Shieh, and J. Zhang, Sci. Rep. 2014, 4, 6491.

[16] E. Laux, C. Genet, T. Skauli and T. W. Ebbesen, Nat. Photonics 2008, 2, 161.

[17] T. Xu, Y. Wu, X. Luo and L. Guo, Nat. Commun 2010, 1, 1058.

[18] M. Tseng, J. Yang, M. Semmlinger, C. Zhang, P. Nordlander and N. Halas, Nano Lett. 2017, 17, 6034 .

[19] N. L. Dmitruk, M. Klopeleisch, O. I. Mayeva, S. V. Mamykin, E. F. Venger and O. B. Yastrubchak, Phys. Stat. Sol.(a) 2001, 184, 165.

[20] F. M. Tuffy, Proceedings of SPIE 2000, 3973, 231.

[21] T. Velten, F. Bauerfeld, H. Schuck, S. Scherbaum, C. Landesberger and K. Bock, Microsyst. Technol. 2011, 17, 619.

[22] S. Song, X. Ma, M. Pu, X. Li, K. Liu, P. Gao, Z. Zhao, Y. Wang, C. Wang and X. Luo, Adv. Opt. Mater. 2017, 5, 1600829.

[23] Szeghalmi, M. Helgert, R. Brunner, F. Heyroth, U. GÖsele and M. Knez, Adv. Funct. Mater. 2010, 20, 2053.

[24] T. Sondergaard and S. I. Bozhevolnyi, Opt. Express 2007, 15, 4198.

[25] D. C. Skigin and R. A. Depine, Phys. Rev. Lett. 2005, 95, 217402.

[26] Z. C. Ye, J. Zheng, S. Sun, L. D. Guo and H. P. D. Shieh, IEEE J. Sel. Top. Quant. Elec. 2013, 19, 4800205.

[27] D. Wan, H. Chen, Y. Lai, H. Yu and K. Lin, Adv. Funct. Mater. 2010, 20, 1742. 


\section{WILEY-VCH}

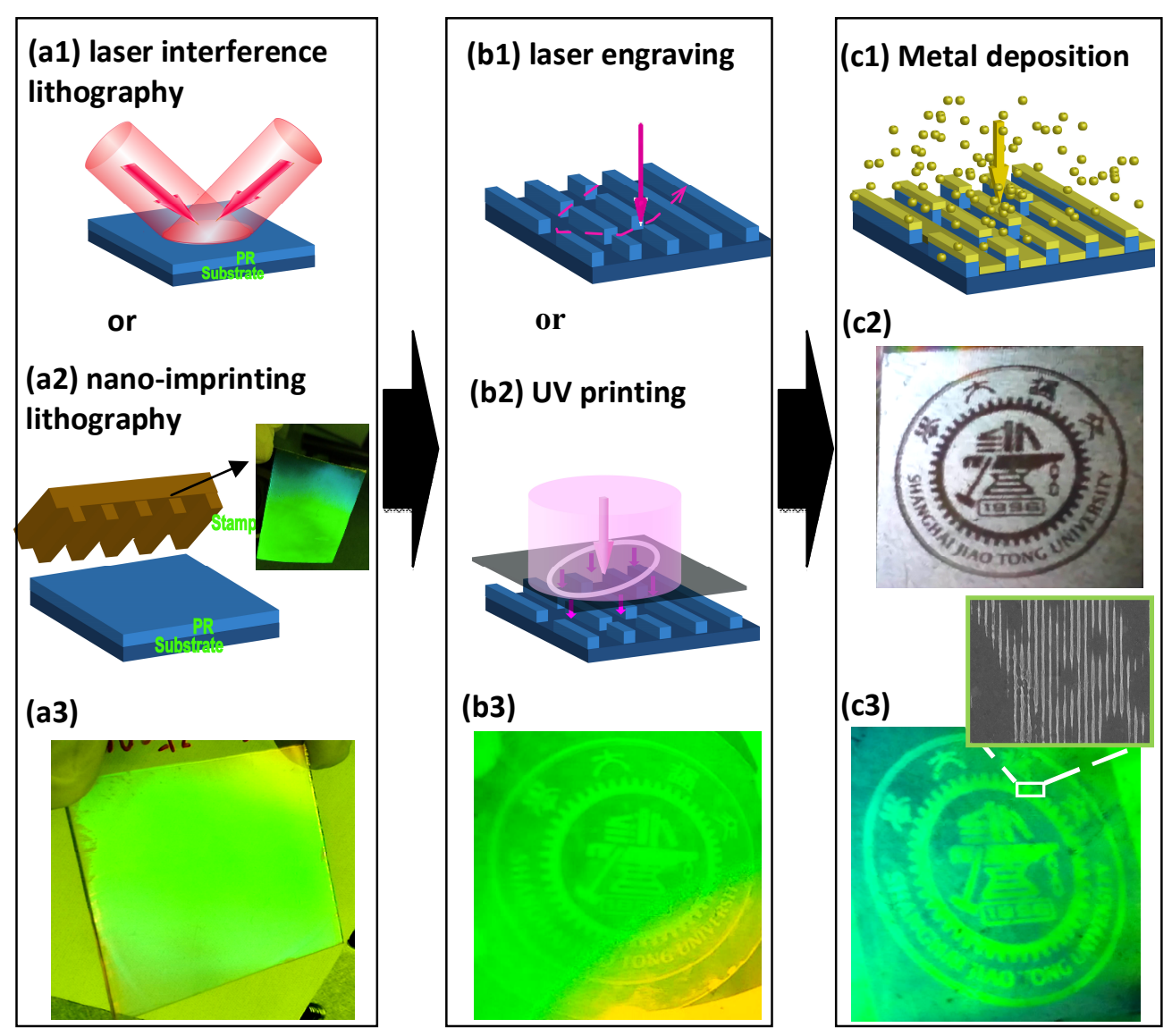

Figure 1. The Fabrication procedure of the proposed MNG-based PCPT. Firstly, dielectric grating templates are fabricated by using LIL (a1) or NIL (a2). A diffractive snapshot of a nickel stamp is presented in (a2). Then, the figures of tags are patterned on the templates by using direct mask-free laser engraving (b1) or rapid mask-based UV printing (b2), where only gratings in the figures are kept. Finally, by depositing appropriate thickness of $\mathrm{Al}(\mathrm{c} 1)$, the demanded colors of the plasmonic tags are obtained. A reflective snapshot of the final PCPT is presented in (c2). Diffractive snapshots of the fabricated samples in each step are presented in (a3, b3, c3). A SEM image is presented in (c3) as well, which clearly displays that only gratings are remained in the figures. 

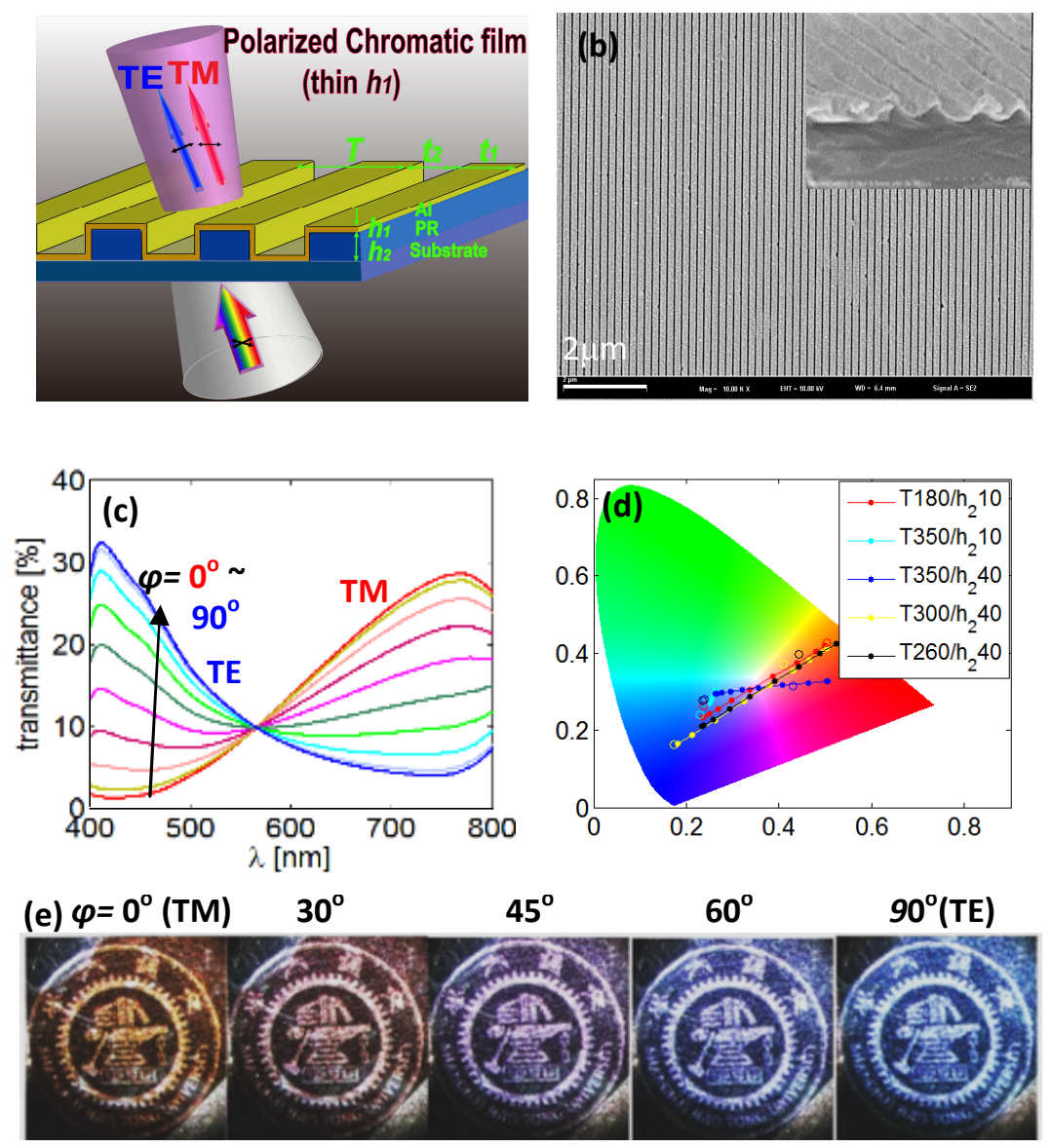

Figure 2. The structure and measured transmission of the fabricated MNG-based PCPTs. (a) The schematic diagram of the MNG. (b) The top and side (inset) SEM images of the fabricated MNGs with pitch of $180 \mathrm{~nm}$. (c) Polarization-dependent transmitted spectra with light incident angle $\theta=0^{\circ}$ and the polarization angle $\varphi$ from $0^{\circ}$ (TM, red line) to $90^{\circ}$ (TE, blue line) at a step of $10^{\circ}$. (d) The spectra's corresponding points in the CIE 1931 xy chromaticity diagram. The measured (the color lines with solid dots) and simulated (the circles) color shifts of the devices with the variation of light polarizations under different grating pitch $T$ and $\mathrm{Al}$ thickness $h_{1}$. The red and other color points correspond to the spectra in (c) and Figure 5(a), respectively. (e) Snapshots of the fabricated PCPT illuminated by white light with different polarization state. represent 


\section{WILEY-VCH}

(a)

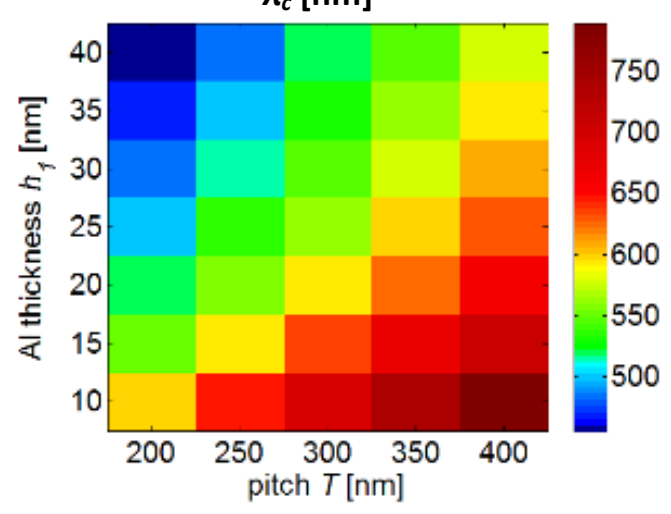

(b)

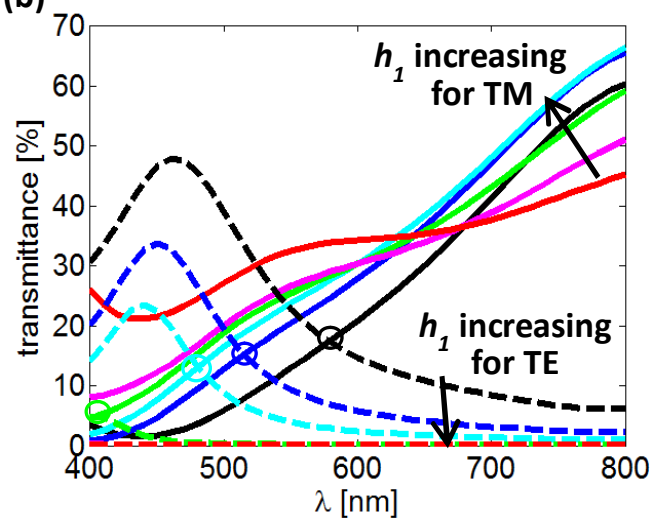

Figure 3. Dependence of $\lambda_{c}$ on Al thickness $h_{1}$ and grating pitch $T$. (a) Simulation results of $\lambda_{c}$ varying with $h_{1}$ and $T$, where dielectric thickness $h_{2}=130 \mathrm{~nm}$ and width $t_{l}=T / 2$. (b)

Calculated TM (solid lines) and TE (dashed lines) transmittance for the grating with $h_{l}=10$, $15,20,40,60$, and $80 \mathrm{~nm}$. The circle markers indicate $\lambda_{c}$. Other parameters are the same with that in Figure 2.

(a)

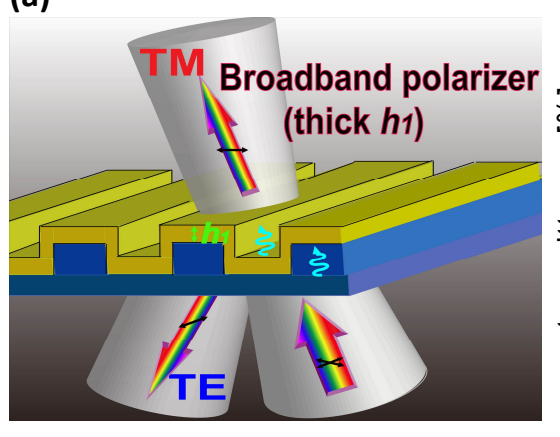

(b)

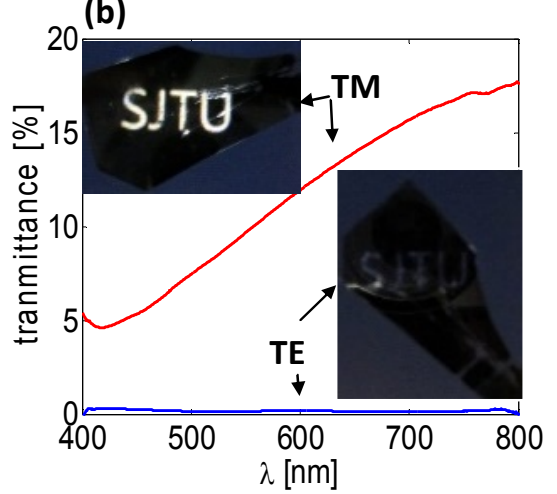

Figure 4. PCPT with a thick Al layer. (a) The schematic diagram of the MNG acting as a broadband polarizer. The blue wave curves in the PCPT represent the longitudinal plasmonic slit-waveguide mode, which is the primary physical component responsible for the polarization dependent transmission. (b) The measured TM (red line) and TE (blue line) transmissions for a grating with $\mathrm{Al}$ thickness $h_{l}=60 \mathrm{~nm}$, and the other parameters are the same with those in Figure 2(b). The snapshots illustrate the transmitted bright TM and dark TE polarized colors of the characters of "SJTU" composed of MNGs. 


\section{WILEY-VCH}

(a)
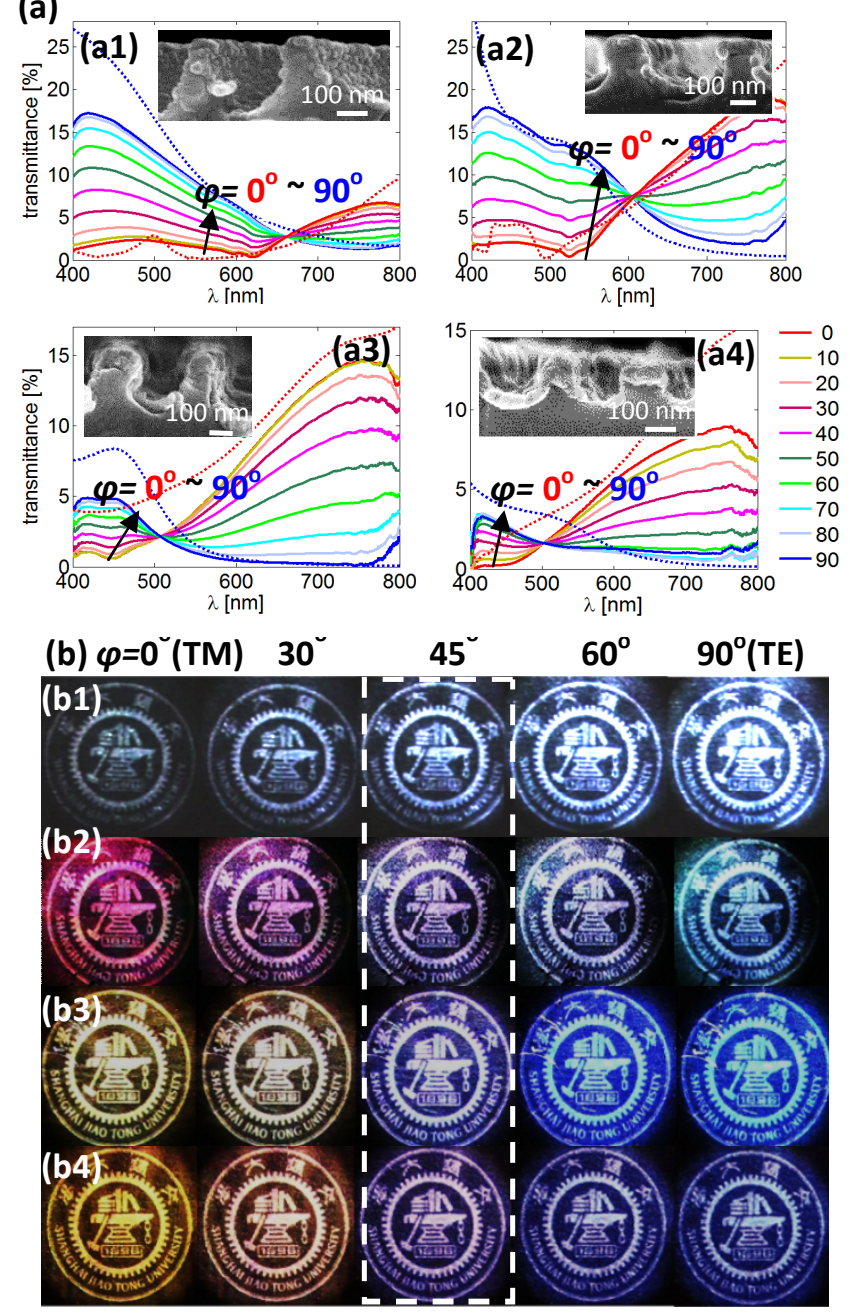

Figure 5. Polarization-dependent transmission measured experimentally with different Al thickness $h_{1}$ and grating pitch $T$. (a) Transmission spectra with polarization angle $\varphi$ varying from $0^{\circ}$ to $90^{\circ}$ at a step of $10^{\circ}$. The solid lines are experimental results. The blue and red dashed lines are the simulated results for $\varphi=0^{\circ}$ and $90^{\circ}$, respectively, whose intensity are divided by 2 for clear comparison with the experimental results. The insets are the side SEM images of the PCPTs. (b) The snapshots of PCPTs for different polarization states, where the pictures within the dashed rectangle are the same with those observed under unpolarized light. Following are the corresponding parameters for the PCPTs: (a1, b1) $T=350 \mathrm{~nm}, h_{1}=15 \mathrm{~nm}$; (a2, b2) $T=350 \mathrm{~nm}, h_{l}=40 \mathrm{~nm}$; (a3, b3) $T=300 \mathrm{~nm}, h_{l}=40 \mathrm{~nm}$. (a4, b4) $T=260 \mathrm{~nm}, h_{l}=40$ $\mathrm{nm}$. The dielectric thickness $h_{2}=150 \mathrm{~nm}$ and width $t_{1}=0.5 T$. 


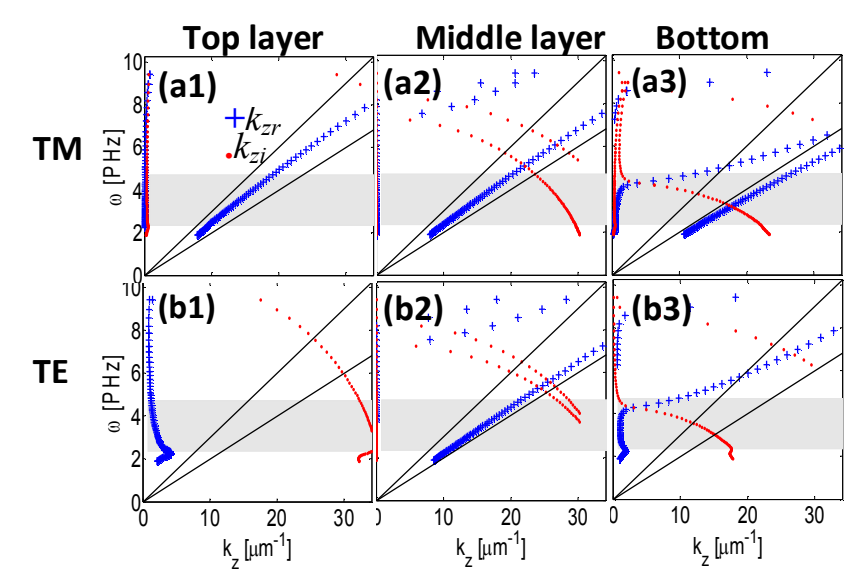

Figure 6. Dispersion curves of the waveguides in three layers of the MNGs: (a1, b1) the airAl-air grating in the top layer, $(\mathrm{a} 2, \mathrm{~b} 2)$ the air-PR-air grating in the middle layer and $(\mathrm{a} 3, \mathrm{~b} 3)$ the Al-PR-Al grating in the bottom layer, where (a1-a3) are for TM light and (b1-b3) are for TE light. The black lines represent the PR and air lines. The gray regions highlight the visible light zone. Simulation parameters are the same as that in Figure 2.

(a) $h_{1}=10 \mathrm{~nm}$

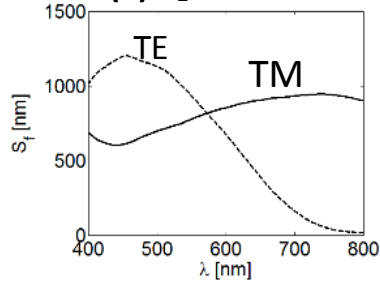

(b) $h_{1}=60 \mathrm{~nm}$

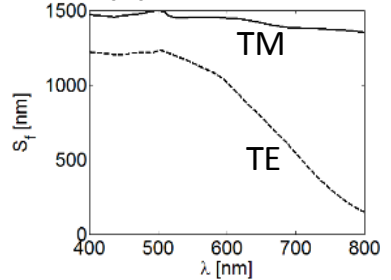

Figure 7. The forward scattering cross-section $\left(S_{f}\right)$ for pitch $T=180 \mathrm{~nm}$ and Al thickness (a) $h_{l}=10 \mathrm{~nm}$ and (b) $h_{l}=60 \mathrm{~nm}$. Other simulation parameters are the same as that in Figure 2.

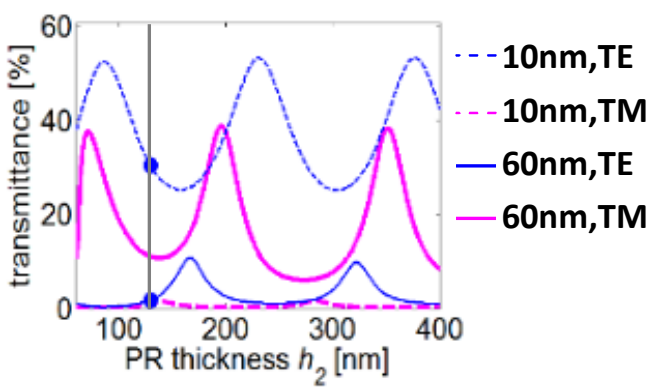

Figure 8. Transmittance varying with PR thickness obtained from simulaiton. The transmittance for TM (solid lines) and TE (dashed lines) light with wavelength of $400 \mathrm{~nm}$ in case of pitch $T=180 \mathrm{~nm}$ and Al thickness $h_{l}=10 \mathrm{~nm}$ (blue lines) and $60 \mathrm{~nm}$ (violet lines). The black vertical line represents the case in our fabricated device with the PR thickness $h_{l}=130$ $\mathrm{nm}$. Other simulation parameters are same with that in Figure 2. 


\section{WILEY-VCH}

The table of contents entry should be 50-60 words long, and the first phrase should be bold. The entry should be written in the present tense and impersonal style. The text should be different from the abstract text.

Keywords: plasmonic structural colors, sub-wavelength grating, metallic nanowire gratings, polarizer, color filter

Jun Zheng*, Zhi-Cheng Ye, Cheng-Liang Wang, Yi-Fei Fu, Xin-Ran Huang and Zheng-Ming Sheng

Highly tunable polarized chromatic plasmonic films based on sub-wavelength grating templates

ToC figure ((Please choose one size: $55 \mathrm{~mm}$ broad $\times 50 \mathrm{~mm}$ high or $110 \mathrm{~mm}$ broad $\times 20 \mathrm{~mm}$ high. Please do not use any other dimensions))

A polarized chromatic plasmonic film is proposed based on sub-wavelength grating structure, which enables 'blue transmission' for the transverse electric (TE) light and 'red transmission' for the transverse magnetic (TM) light. Based upon the unique transmission spectrum characteristics of such films, polarized chromatic plasmonic tags (PCPTs) with demanded versatile polarized transmission colors can be fabricated in a large scale.

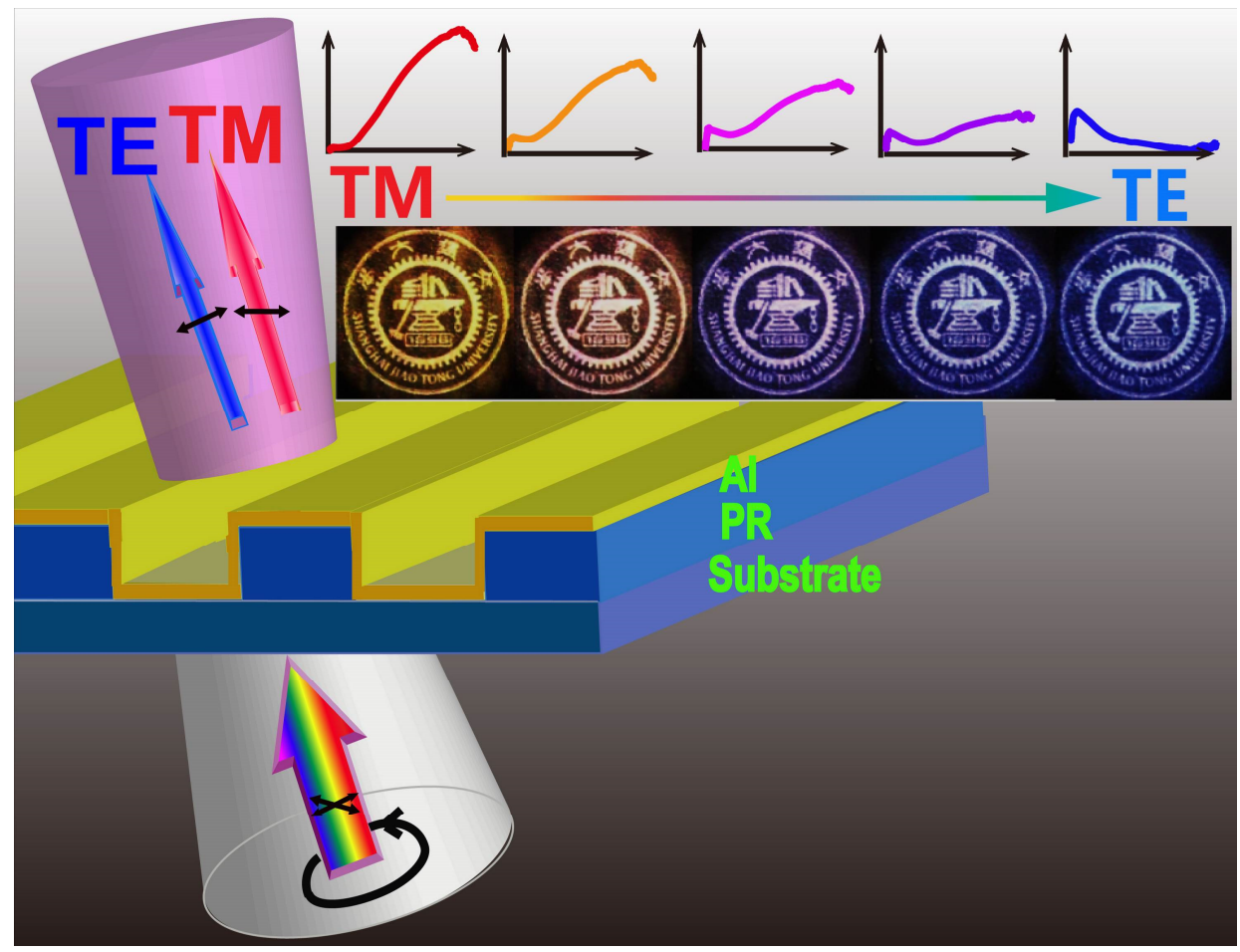

